\title{
Elementos para la valoración integral de proyectos de emprendimiento social. Una herramienta para la formación de emprendedores
}

\section{José Carlos Vázquez Parra}

Escuela de Humanidades y Educación

Tecnológico de Monterrey en Guadalajara

Cuando se alude a la formación de emprendedores, se suele pensar de manera casi automática en cuestiones relacionadas con las ciencias administrativas o las escuelas de negocios; sin embargo, el emprendimiento tiene ciertas variables con objetivos mucho más amplios y con un enfoque primordialmente social. El emprendimiento social es el desarrollo de un proyecto innovador con el que se busca solventar o contribuir a un problema social, como puede ser la pobreza, la desigualdad o el desempleo, a partir de un modelo de negocio de carácter inclusivo. El presente artículo propone, a partir de un análisis de contenidos de textos académicos del área, la necesidad de considerar cinco elementos (normatividad, profesionalismo, inclusión, valor compartido y sustentabilidad) para la valoración integral de los proyectos de emprendimiento social. Se concluye que estos pueden ser una excelente herramienta que pueda guiar la formación y las decisiones de los futuros emprendedores sociales al abrir la necesidad de incluir factores que usualmente no se consideran en la formación de emprendedores tradicionales.

Palabras clave: emprendimiento social, formación de emprendedores, inclusión, valor compartido.

\section{Elements for the integral valuation of social entrepreneurship projects. A tool for the training of entrepreneurs}

When we talk about training entrepreneurs, we usually think almost automatically about issues related to administrative sciences or business schools, however, entrepreneurship has certain variables with much broader objectives, with a primarily social focus. Social entrepreneurship is the development of an innovative project that seeks to solve or contribute to a social problem, such as poverty, inequality or unemployment, based on an inclusive business model. This article proposes the need to consider five elements (normativity, professionalism, inclusion, shared value and sustainability) for the integral assessment of social entrepreneurship projects, considering that these can be an excellent tool that can guide training and decision-making of future social entrepreneurs, by opening the need to consider factors that are usually not considered in the training of traditional entrepreneurs.

Keywords: Social entrepreneurship, training of entrepreneurs, inclusion, shared value. 


\section{Elementos para a valorizaçáo integral de projetos de empreendedorismo social. Uma ferramenta para a formação de empreendedores}

Quando falamos de formação de empreendedores, geralmente pensamos quase automaticamente em questóes relacionadas a ciências administrativas ou escolas de negócios, no entanto, o empreendedorismo tem certas variáveis com objetivos muito mais amplos, com um foco principalmente social. Empreendedorismo social é o desenvolvimento de um projeto inovador que busca resolver ou contribuir para um problema social, como pobreza, desigualdade ou desemprego, baseado em um modelo de negócios inclusivo. Este artigo propóe a necessidade de considerar cinco elementos (normatividade, profissionalismo, inclusão, valor compartilhado e sustentabilidade) para a avaliaçáo integral dos projetos de empreendedorismo social, considerando que estes podem ser uma excelente ferramenta que pode orientar o treinamento e a tomada de decisáo. futuros empreendedores sociais, abrindo a necessidade de considerar fatores que normalmente não são considerados na formação de empreendedores tradicionais.

Palavras-chave: empreendedorismo social, formação de empreendedores, inclusão, valor compartilhado.

\section{Introducción}

Emprender es más que una actitud: es una aptitud de las personas que les permite planear, organizar y arrancar nuevos retos y proyectos, que inicia un caminar constante hacia sus objetivos (Marulanda y Morales, 2016). Emprender es desarrollar ideas que persiguen un cierto fin, que puede ser de tipo económico, político, social, entre otros. Hoy en día, se habla mucho de la necesidad de emprender — sobre todo, en los ámbitos universitarios de formación profesional-, en la medida que se considera que un liderazgo sin emprendimiento es como tener una buena idea sin conseguir aterrizarla (Rodríguez, 2016).

Aparejado al esfuerzo de las instituciones educativas, los gobiernos latinoamericanos parecen estar prestando mucha atención al emprendimiento, y han puesto en marcha diversos proyectos para el fomento y la promoción de las ideas de los emprendedores de la región (García, Gracia y Días, 2015). Así, nuevas nociones parecen incluirse en el lenguaje de las organizaciones, considerando aspectos como la innovación de carácter empresarial, la generación de células e incubadoras de negocios, así como el impacto que un buen sistema de coaching puede aportar a la generación de nuevas empresas. La apuesta de la economía latinoamericana hacia las micro, pequeñas y medianas empresas (MiPyMes) parece venir acompañada de una promoción y un respaldo de los modelos de emprendimiento (García et al., 2015).

Sin embargo, no se puede asumir que el emprendimiento está únicamente motivado por fines económicos. De hecho, en la última década, ha sido cada vez más usual encontrarse con proyectos de emprendimiento que tienen como objetivo contribuir a resolver una problemática social o humana (Padilla, Quispe, Nogueira y Hernández, 2016). Esto es lo que se denomina «emprendimiento social», un modelo de emprendimiento que busca soluciones innovadoras para resolver problemas existentes de una sociedad, como lo es la pobreza, el hambre, el desempleo, la discriminación, el impacto en el medio ambiente, entre otros, para impulsar así el desarrollo de la comunidad (Naranjo, 2015). A diferencia del emprendedor tradicional, quien tiene fines de lucro, los emprendedores sociales tienen a una generación 
de valor social. Según Palacios (2010), el emprendimiento social suele funcionar a través de empresas sociales que, por medio del uso de fondos públicos o privados, generan un cambio social desarrollando actividades, programas, proyectos o servicios detonantes de valor compartido.

Lamentablemente, de acuerdo con Stewart (2002), solo el $40 \%$ de las iniciativas sociales superan los 5 años de vida tras su fundación. Aunque es el doble del rango de supervivencia de las MiPyMes de la región, continúa siendo un parámetro altamente desafiante para los emprendedores. Por ende, las instituciones de formación deben atender a los diferentes factores que pueden verse implicados entre el fracaso o la supervivencia de los emprendimientos sociales, puesto que los recursos económicos y humanos invertidos terminan significando una pérdida a mediano y largo plazo si la empresa no consigue continuar después de unos años (Sepúlveda y Gutiérrez, 2016).

Fernández, Revuelto y Simón (2018), en su estudio «Supervivencia de empresas sociales de nueva creación. Un enfoque basado en el análisis cualitativo comparativo fsQCA», llegan a la conclusión de que no es posible identificar alguna condición concreta por sí misma, debido a que existen diversas soluciones suficientes, según las características y la madurez de las empresas, para evitar su cierre. Sobre esta base, el presente artículo plantea una serie de elementos para la valoración integral de proyectos de emprendimiento social, los mismos que pueden constituirse como parámetros a ser considerados dentro de la formación de nuevos emprendedores en las instituciones educativas. A partir de estos cinco elementos clave —normatividad, profesionalismo, inclusión, valor compartido y sustentabilidad-, se pretende replantear la manera en que se vienen constituyendo los emprendimientos sociales, de modo que estos sean más integrales; que estén acordes con la realidad; $y$, por ende, que tengan mayores posibilidades de supervivencia.

\section{El emprendimiento social: una nueva visión en la formación de emprendedores}

La actividad emprendedora es una gestión de cambio radical, discontinua y disruptiva que renueva, de manera estratégica, la visión que tenemos de las cosas (Álvarez y Rodríguez, 2015). De manera adicional a estas características, el emprendimiento social resalta la conciencia en cuanto a una visión sistémica de las organizaciones y los negocios, considerando la manera en que los agentes se relacionan, interactúan e influyen unos en otros a partir de proyectos conjuntos. El emprendimiento social, del mismo modo que la responsabilidad social, buscan la generación de valor compartido velando por el bienestar y el desarrollo de los emprendedores, así como de todo agente que le rodea y de la sociedad en la que se encuentran (Vega y Mera, 2016).

Sin embargo, la constitución de este nuevo modelo de emprendimiento, que difiere claramente de aquel que tradicionalmente veía de manera exclusiva por el lucro económico, implica la necesidad de una formación de emprendedores distinta. Debe ser una formación en que la motivación social y el valor compartido sean una parte fundamental de lo que se comparte y espera de ellos. Esta situación ha llevado a que diferentes universidades estén apostando por una nueva manera de plantear sus programas de negocios, en los que el emprendimiento social tenga un espacio concreto en la currícula de sus estudiantes, $y$, de manera adicional, se fomenten competencias de compromiso y responsabilidad social, así como de colaboración, inteligencia social y desarrollo comunitario. 


\section{El estudio Alfa-Tuning Latin American Project} 2011-2013 presenta resultados de 7 programas iberoamericanos que incluyen la formación en emprendimiento social, lo cual se suma a una visión de innovación social universitaria responsable (Sáenz y López, 2015). En este estudio, encabezado por la Universidad de Deusto, en España, participaron 15 universidades, tanto de América Latina, como de España y Dinamarca (Sáenz y López, 2015). A partir de las conclusiones presentadas, se observa la necesidad de que la formación de emprendedores sociales se dé con una visión, aunque semejante a otros tipos de emprendimiento, que incluya aspectos adicionales, como es un claro rumbo humano, el desarrollo de habilidades sociales y un fuerte sentido ciudadano. La idea es que los emprendedores sociales puedan comprender el contexto social, económico, político y cultural de los problemas que están tratando de resolver, es decir, que incorporen una visión externa al proyecto o a la organización propuesta (Nikulin, Viveros, Dorochesi, Crespo y Lay, 2017).

Un proyecto semejante es el Programa de Fomento a la Actitud Emprendedora en México, que incluye a la Asociación Nacional de Universidades e Instituciones de Educación Superior (Anuies), así como a la Fundación Educación Superior-Empresa (FESE). Este programa no solo considera la relevancia de aspectos administrativos y contables al momento de emprender, sino también la importancia de darle protagonismo a la función emprendedora como instrumento de movilidad social (Hernández, 2015). A este proyecto se le suma Emprendimiento Social MX. Esta es una organización de entidades sociales que buscan impulsar el talento de los universitarios para diseñar soluciones a problemas sociales a través del fomento y de la formación para la generación de emprendimiento, y de la vinculación para aumentar el impacto social (Esmex, 2017).
Otro ejemplo es el programa «Aprender a Emprender», proyecto aplicado en Perú, que busca capacitar a jóvenes emprendedores bajo una visión de apertura a las problemáticas de su entorno. El objetivo es que, a partir de la generación de empresas innovadoras, puedan plantear soluciones a alguno de los problemas de la región (Oliver, Galiana y Gutiérrez, 2016).

Como se puede apreciar con estos ejemplos, el emprendimiento social se ha convertido en una parte natural de la formación de emprendedores: ha pasado de ser una alternativa a una parte de las consideraciones que debe considerar cualquier nuevo proyecto educativo del área de negocios. En países como México, se pueden encontrar múltiples proyectos de emprendimiento social (Tabla 1), que reflejan la importancia de la formación de emprendedores sociales, puesto que estos han surgido de los diferentes programas estatales o universitarios de fomento al emprendimiento.

De esta forma, se puede constatar la importancia de la formación de emprendedores sociales, los cuales, más allá de generar un negocio, promueven el desarrollo de las comunidades de una forma eficiente, inclusiva y no asistencialista (Ashoka, 2017). Sin embargo, no basta con invitar a los emprendedores a cambiar su motivación lucrativa por una de corte social, sino que es necesario darles herramientas para generar verdaderos proyectos de impacto y que, de manera integral, fomenten el bienestar de la comunidad.

Por consiguiente, las instituciones de educación superior deben proveer de esta nueva visión a la formación que actualmente dan en las áreas de negocios considerando lo altamente conveniente que resulta el fomento de emprendimiento social en regiones en desarrollo como la latinoamericana. 


\section{Tabla 1. Proyectos de emprendimiento social en México surgidos de instituciones educativas}

\begin{tabular}{|c|c|}
\hline Proyecto & Objetivo \\
\hline Yo propongo & $\begin{array}{l}\text { Construye canales de participación ciudadana inclusivos a través del desarrollo de herramientas tecnológicas inno- } \\
\text { vadoras. }\end{array}$ \\
\hline Lab4U & $\begin{array}{l}\text { A través de smartphones, ofrece a las escuelas herramientas para enseñar ciencias a los niños y las niñas a través de } \\
\text { divertidos experimentos. }\end{array}$ \\
\hline SVX & Trabaja con inversionistas, fondos, bancos e instituciones financieras enfocadas en la inversión de impacto social. \\
\hline Inclúyeme.com & Es la primera bolsa laboral incluyente de América Latina. \\
\hline Laboratoria & $\begin{array}{l}\text { Es una empresa que empodera a otras mujeres enseńándoles programación, código y desarrollo para que puedan } \\
\text { acceder por primera vez a oportunidades laborales formales. }\end{array}$ \\
\hline Misión Planeta & $\begin{array}{l}\text { Diseńa programas para trabajar y vincular diversos sectores de la sociedad, adaptando cada proyecto para así lograr el } \\
\text { mayor impacto social, ambiental y económico posible. }\end{array}$ \\
\hline Protrash & $\begin{array}{l}\text { Esta empresa social se dedica a optimizar la industria del reciclaje en México y, al mismo tiempo, a ayudar a las per- } \\
\text { sonas de comunidades de bajos ingresos a adquirir un valor monetario por sus residuos reciclables. }\end{array}$ \\
\hline Sarape Social & $\begin{array}{l}\text { Esta agencia de publicidad usa el poder de las personas, las historias, la creatividad, los medios y la tecnología para } \\
\text { hacer del mundo un mejor lugar. Sus campañas de comunicación concientizan, informan y promueven valores } \\
\text { impulsando proyectos e iniciativas de empresas y gobiernos. }\end{array}$ \\
\hline Tu Ola & $\begin{array}{l}\text { Es el primer programa en México que recompensa a los usuarios por participar en acciones que transformen su } \\
\text { entorno de manera positiva. }\end{array}$ \\
\hline EcoTierra & $\begin{array}{l}\text { Es una organización campesina del istmo de Tehuantepec Oaxaca en el sureste de México que siembra y cosecha } \\
\text { diversos cultivos como el ajonjolí, el tamarindo, el chile pasilla navideño con el objetivo de desarrollar a los miembros } \\
\text { de la comunidad con igualdad, participación, respeto al ambiente y a la cultura. }\end{array}$ \\
\hline
\end{tabular}

Fuente: Ecotierra (2017), Inadem (2017), Misión Planeta (2016), ProTrash (2017), Sarape Social (2016), Tu Ola (2016).

\section{Elementos para la valoración integral de proyectos de emprendimiento social}

La formación de emprendedores suele venir aparejada por una fuerte carga de información y capacitación de varias índoles, la cual suele considerar el desarrollo de competencias administrativas, financieras y contables (García, 2015). Sin embargo, frente a los modelos de emprendimiento social, los jóvenes emprendedores requieren de habilidades que también desarrollen en ellos una visión transversal del mundo, que les permita considerar todas las aristas que pueden relacionarse con un proyecto social.
Ello, sin lugar a duda, puede impactar en la planeación, la fundación y el desarrollo de un proyecto de emprendimiento, puesto que cualquier decisión, sin la información pertinente, puede no considerar algún aspecto que sea la diferencia entre el fracaso y la supervivencia (Contreras, Wilches, Graterol y Bautista, 2017).

Por consiguiente, el trabajo de las instituciones educativas y los docentes ya no basta para desarrollar proyectos de innovación de carácter empresarial; hoy en día, la sociedad espera más de estos programas formativos (Munster, 2017). El impulso del emprendimiento social debe acompañarse de una visión 
de futuro que dé pauta a ideas creativas, eficientes, responsables y efectivas (González y Fernández, 2015). Así, aspectos como la sustentabilidad, el consumo responsable, la perspectiva de grupos de interés, la tendencia de mercados inclusivos y/o la responsabilidad social son elementos que resultan diferenciadores entre el emprendimiento social y aquel de corte tradicional, y que apoyan a plantear acciones acordes con las agendas sociales internacionales.

Como respuesta a esto, en el ámbito educativo, se han generado variadas propuestas de innovación educativa en torno a este tema, como lo son los trabajos del Centro de Iniciativas Emprendedoras de la Universidad Autónoma de Madrid o el modelo de formación de emprendimiento social propuesto por Vega y Mera (2016) de la Universidad Nacional de Colombia. Estas propuestas pretenden relacionar la formación de cada estudiante con proyectos de emprendimiento que incluya un nutrido apartado disciplinar, con pinceladas profundas de creatividad y originalidad, así como con un claro sentido humano y responsabilidad social. Sin embargo, aunque lo anterior pudiera parecer sencillo, trae consigo un nivel de reflexión que muchas veces no se consigue, debido a que, a diferencia del emprendimiento tradicional, el emprendimiento social implica competencias como el reconocimiento, la empatía o la capacidad de juicio que no siempre pueden trabajarse dentro de un salón de clases (Vázquez, 2017).

En este sentido, se plantea la necesidad de considerar ciertos elementos como una parte fundamental de la constitución y la propuesta de proyectos de emprendimiento social, los mismos que se incluyen como parámetros necesarios en la formación de nuevos emprendedores. Es prioritario que los emprendedores consigan desarrollar competencias sociales que se sumen a las habilidades disciplinarias. Aunque esto no puede asegurarse, sí debe considerarse en la gestación de nuevos proyectos. Por ello, el presente artículo plantea cinco elementos de valoración de proyectos que todo emprendedor debería considerar, en la medida que se constituyen como un primer paso a tomar en cuenta en la preparación de propuestas sociales.

Para poder determinar estos elementos, los investigadores hemos realizado un análisis de contenido de variadas fuentes académicas - las que se reflejan en la explicación posterior-. De este modo, se ha buscado dar un marco de referencia que sirva a los nuevos emprendedores sociales al momento de estructurar sus propuestas. A partir de estos cinco elementos —normatividad, profesionalismo, inclusión, valor compartido y sustentabilidad-, se apunta a marcar una pauta en la estructuración de proyectos de emprendimiento social. Se espera que dichos elementos se planteen como una necesidad que debe considerarse al momento de formar nuevos emprendedores (Figura 1).

Figura 1. Elementos para la valoración integral de proyectos de emprendimiento social

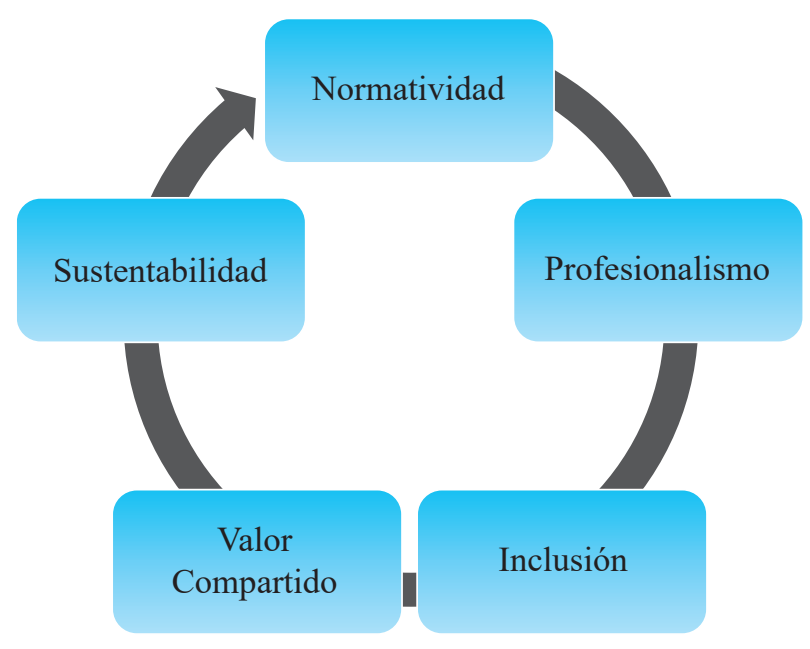


Como se ha señalado anteriormente, estos elementos se plantean como componentes que deben considerarse al momento de proponer un proyecto de emprendimiento social, en la medida que son factores que garantizan que dicha propuesta sea más integral; esté en sintonía con el sentido inicial de generación de valor social compartido; y que, por ende, brinde mayor estabilidad y continuidad al proyecto. A continuación, se profundiza en los mismos.

\subsection{Normatividad}

La normatividad hace referencia a la necesidad de que el proyecto se ajuste y constituya basándose en la legislación y la normatividad pertinentes, tanto a nivel administrativo como del giro o rumbo que se espera tome a mediano y largo plazo. No se puede evitar pensar que cualquier proyecto de emprendimiento está suscrito a un espacio y a un tiempo, y, como consecuencia, debe adecuarse a lo que en dicha temporalidad se considera pertinente, correcto y legal. La normatividad debe incluir todo lo relacionado con lo administrativo, lo contable y lo operativo, marco en el cual se debe tomar en cuenta aquellas normas que deban aplicarse en la generación como en el desarrollo de la propuesta (Vásquez y Dávila, 2008). En el caso de emprendimientos que tengan la posibilidad de desarrollarse internacionalmente, es importante que los emprendedores cuenten con la orientación legal adecuada para que su proyecto no se vea afectado por este tema normativo.

\subsection{Profesionalismo}

El segundo elemento a considerar se relaciona con el profesionalismo, es decir, con el desarrollo de los valores propios de las personas que intervienen en el proyecto, así como los de sus profesiones. Un punto que usualmente afecta al emprendimiento social es la falta de conocimientos o identificación de los emprendedores con el giro u objetivo del proyecto, o peor aún, la falta de convicción de la labor que se realiza (Contreras et al., 2017). Para conseguir un verdadero profesionalismo, los programas formativos deben evaluar la manera en que el perfil de los emprendedores sea el más acorde a la propuesta que se realiza, y, en caso de no ser así, la manera de capacitarlos en dichos rubros y competencias. Cabe señalar que el ser emprendedor no necesariamente obliga a ser un profesional del área económico-administrativa, por lo que depende de las instituciones de formación brindar las herramientas, las competencias y las habilidades para que el proyecto tenga las mayores posibilidades de desarrollarse de manera eficiente (Contreras et al., 2017).

\subsection{Inclusión}

Un aspecto que debe caracterizar a cualquier proyecto de emprendimiento social es el carácter inclusivo, es decir, el considerar a todos aquellos que podrían verse afectados, beneficiados o relacionados con lo que se pretende hacer. $\mathrm{Al}$ respecto, es relevante que los emprendedores tengan mucha apertura hacia la generación de modelos de negocios inclusivos, es decir, modelos que busquen mejorar las condiciones de vida de personas que usualmente se encuentran en la base de la pirámide económica o que son segregados de la misma (Vásquez y Dávila, 2008). Cabe señalar que lo inclusivo debe articularse con lo incluyente, lo cual implica que los proyectos de emprendimiento social deben cuestionarse acerca de los grupos de interés que se están considerando al momento de la planeación y el desarrollo de la propuesta. Por ende, se espera que la formación de los nuevos emprendedores incluya la adquisición de claras habilidades de reconocimiento de las necesidades de los otros que los rodean y lo que para ellos resulta valioso e importante (Moreira y Urriolagoitia, 2011). Una 
visión incluyente de personas con alguna discapacidad, una perspectiva de género, o una consideración a cuestiones indigenistas o de las comunidades LGBTI+ debe formar parte de un proyecto de emprendimiento social (Ortiz, 2017).

\subsection{Valor compartido}

De manera complementaria a la inclusión, y al seguimiento de las normas y las leyes, los proyectos de emprendimiento social deben tener la apertura para considerar aquello que para los grupos de interés es importante y adecuado; en ese sentido, es responsabilidad de los emprendedores identificar, incluir y poner atención a las legítimas demandas de los agentes afectados o relacionados (Romero, Hernández, Gutiérrez y Portillo, 2017). Un problema usual que se da con la filantropía es que suele ser de tipo muy general; es decir, pretende poder impactar a diferentes comunidades con situaciones semejantes. Esto conduce a que muchas veces los apoyos no lleguen ser los adecuados para ciertos grupos; de este modo, se limitan a ser una ayuda valiosa, pero no verdaderamente eficiente. Este también podría ser el escenario de los proyectos de emprendimiento social si no se cuenta con una exploración previa que tenga como intención conocer a sus grupos de interés (Palacios, 2010). Acciones como identificar necesidades, valores o creencias pueden ser la diferencia entre ofrecer algo sostenible a mediano y largo plazo, o quedarse únicamente con una ayuda a un problema concreto desde una visión externa al mismo. La idea de generar valor compartido se sostiene en el hecho de que el proyecto de emprendimiento debe considerar las diferentes vertientes de valor con la intención de no verse limitado sobre lo que busca alcanzar. A continuación, se detallan las vertientes de valor que se consideran relevantes en lo que respecta a un verdadero valor compartido: d.1. Valor económico: Hace referencia a la generación de recursos de carácter económico que genera el proyecto. Hay que considerar que el emprendimiento social tiene una base económica, por lo que, aunque el objetivo del mismo sea apoyar o resolver un problema social o humano, el tema de los recursos económicos es relevante para garantizar la permanencia y el desarrollo del proyecto (Carona, Dinora y Tabares, 2008). Es preciso anotar que, pese a que el emprendimiento social debe velar por un desarrollo económico, su objetivo no debe enfocarse en la producción de dinero. Los emprendedores sociales deben contar con las habilidades para lograr un equilibrio entre ambos elementos (Vásquez y Dávila, 2008).

d.2. Valor ambiental: Como parte del desarrollo social que implica este tipo de emprendimiento, se debe considerar el impacto ambiental del mismo. Es relevante que los emprendedores comprendan que el valor compartido supone el aspecto ecológico, puesto que sería poco congruente velar por el bienestar de las personas y, a la vez, afectar negativamente su entorno. En esa medida, el valor ambiental es un punto de suma relevancia (Sanabria y Hurtado, 2018).

d.3. Valor social: Este tipo de valor se relaciona con el sentido mismo del proyecto, es decir, el tener claramente identificado el objetivo o el problema social que se busca solucionar o frente al cual se apunta a contribuir (García et al., 2015). Este es uno de los puntos primordiales y de arranque del proyecto en general.

d.4. Valor de conocimiento: Este punto hace referencia a la capacidad para transmitir y generar nuevo conocimiento a partir del proyecto de emprendimiento. El conocimiento puede ser 
de los mismos emprendedores o bien hacia el exterior, con lo cual se da la posibilidad de que otros puedan en un momento dado replicar o transferir el proyecto en otro espacio (Ocampo, 2016). El valor de conocimiento también puede enfocarse en brindar herramientas a los grupos de interés para hacer que el proyecto se vuelva autónomo o a todo el proceso de aprendizaje, lo que permite mejorar la propuesta inicial (Vázquez, 2017).

d.5. Valor ciudadano: Todo proceso de emprendimiento social debe incluir la contribución al entorno ciudadano del lugar en donde se inserta y desarrolla, puesto que, como se seńalaba en la parte de legislación y normatividad, el proyecto es parte de una realidad de la cual no puede ser indiferente (Vázquez 2017). Por otro lado, cualquier organización, ya sea tradicional o de corte social, debe abonar al desarrollo de la ciudadanía y los valores democráticos, lo cual le obliga a actuar de manera correcta y conforme a los valores ciudadanos, como la transparencia, la legitimidad, la promoción de la gobernanza, entre otros.

\subsection{Sustentabilidad}

Un último elemento es el de la sustentabilidad. Este se enfoca principalmente en la idea de que el proyecto pueda ser sustentable a mediano y largo plazo, lo cual, como se ha planteado en varias partes del artículo, debiera ser uno de los objetivos primordiales de cualquier proyecto de emprendimiento (Romero y Milone, 2016). La idea de considerar la sustentabilidad como un elemento se basa en el principio de que cualquier programa formativo debe tener como uno de sus puntos cardinales dar herramientas, habilidades y competencias a los emprendedores para garantizar, en la medida de lo posible, la sustentabilidad de sus propuestas. Cabe señalar que la sustentabilidad no solo se relaciona con cuestiones ambientales, sino también con el buen aprovechamiento de los recursos económicos, sociales y humanos que implica el proyecto. Para conseguirlo, los emprendedores deben solventar los recursos necesarios para que su visión no se vea afectada por elementos que podrían haberse considerado en la etapa de planeación (Fuentelsaz y González, 2015).

Como se ha señalado anteriormente, y se sustenta en los planteamientos académicos utilizados para su explicación, estos elementos ya han sido considerados por otros artículos, libros y otros textos. Sin embargo, en dichas fuentes, no han sido abordados como factores que requieren incluirse de manera conjunta. Lamentablemente, es usual que las propuestas que surgen en centros universitarios consideren o pongan mayor atención a aquellos elementos en los que se especializan debido al tipo de formación, programas o enfoque de la institución. No obstante, si la idea es poder proyectar el emprendimiento a largo plazo, es necesario que se consideren todos los elementos relacionados, lo que abre la posibilidad a propuestas mucho más integrales y completas (Ocampo 2016).

Uno de los beneficios de gestar una forma emprendimiento que desde su origen incluya o considere elementos de corte económico, social, ambiental y humano es que permite que los mismos tengan mayor posibilidad de supervivencia, en la medida que vela por una totalidad de factores y no se limita al objetivo primordial —el componente social- (Fuentelsaz y González 2015).

\section{Conclusiones}

Cuando se alude a emprendimiento, es usual que hagamos referencia a un sinnúmero de elementos de corte administrativo enfocados en el desarrollo económico 
de la propuesta, como es lo relacionado con el equilibrio financiero, las consideraciones fiscales, los costos de operación, entre otros. Sin embargo, en el caso del emprendimiento social, parece que la visión rotara hacia otros objetivos, como si lo económico no fuera verdaderamente relevante. Lamentablemente, aunque estas dos alternativas pudieran funcionar a corto plazo, el restringir el emprendimiento a lo económico y el emprendimiento social al trabajo comunitario termina menguando de manera profunda las posibilidades de desarrollo y de supervivencia de ambos tipos de proyectos.

Así como sucede con otros modelos de negocios de corte social, como las empresas humanistas o los negocios inclusivos, el emprendimiento social debe buscar un equilibrio entre los diferentes tipos de valor que debe generar, debido a que solo de esta forma podrá proyectarse a mediano y largo plazo. Frente a ello, el presente artículo ha planteado estos cinco elementos que se consideran relevantes para lograr esta proporción y equilibrio entre los valores económicos y sociales del emprendimiento, poniendo especial atención en la necesidad de incluirlos como competencias a desarrollarse en la formación de nuevos emprendedores.

Aunque se reconoce que lo que se presenta aquí es limitado, y que los elementos claramente pueden desarrollarse de una forma más profunda, se considera que el verdadero valor de la propuesta radica en incluir en la discusión académica ciertos puntos que no suelen tomarse en cuenta como una totalidad desde una visión administrativa de la formación de emprendedores. Desde una visión de formación universitaria integral, estos elementos pretenden ser una guía que todo nuevo emprendedor social debiera considerar en aras de tener un enfoque más amplio de la realidad en la que se desenvuelve su proyecto, incluidos algunos aspectos que pudieran comprometer su tasa de supervivencia.

\section{Referencias}

Álvarez, E. y Rodríguez, A. (2015). Inspirando el cambio en educación. Ecosistemas de formación para aprender a emprender. Estudios Pedagógicos, 51, 9-29. https://doi. org/10.4067/S0718-07052015000300002

Ashoka (2017). Ashoka. Recuperado el 4 de junio de 2018, de https://www.ashoka.org/es/stories

Carona, M., Dinora, L. y Tabares, J. (2008). Las dimensiones del emprendimiento empresarial: La experiencia de los programas de cultura del fondo emprender en Medellin [Documentos de trabajo]. Universidad EAFIT, Medellín. Recuperado el 4 de junio de 2018.

Contreras, J., Wilches, S., Graterol, M. y Bautista, M. (2017). Educación superior y la formación en emprendimiento interdisciplinario: un caso de estudio. Formación Universitaria, 10(3), 11-20. https://doi. org/10.4067/S0718-50062017000300003

Ecotierra (2017). Ecotierra. Sabor que da salud. Recuperado el 1 de junio de 2018, de http://ccc-ecotierra.org/

ESMEX (2017). ¿Qué hacemos?. Emprendimiento Social MX. Recuperado el 1 de junio de 2018, de http:// www.emprendimientosocial.mx/

Fernández, R., Revuelto, L. y Simón, V. (2018). Supervivenvia de empresas sociales de nueva creación. Un enfoque basado en el análisis cualitativo comparativo fsQCA. Ciriec-España. Revista de Economía Pública, Socialy Cooperativa, 92, 183-221. https://doi. org/10.7203/ciriec-e.92.10735

Fuentelsaz, L. y González, C. (2015). El fracaso emprendedor a través de las instituciones y la calidad del emprendimiento. Universia Business Review, 47, 64-81.

García, A., Gracia, M. y Días, J. (2015). Emprender en economías emergentes: el entorno institucional y su desarrollo. Innovar. Revista de Ciencias Administrativas y Sociales, 25(57), 133-155. 
García, V. (2015). Emprendimiento Empresarial Juvenil: Una evaluación con jóvenes estudiantes de universidad. Revista Latinoamericana de Ciencias Sociales, Niñez y Juventud, 13(2), 1221-1236. https://doi.org/1 $0.11600 / 1692715 x .13246200315$

González, S. y Fernández, M. (2015). Valores de éxito y emprendimiento. International Journal of Developmental and Educational Psichology, 1(2), 171-184. https:// doi.org/10.17060/ijodaep.2015.n2.v1.333

Hernández, M. (2015). Fomento de la actitud emprendedora, en el ambiente universitario para la creación de MiPyMes. El caso de posgrado de la DACEAUJAT. Hitos de Ciencias Economico Administrativas, 61, 1-16. Disponible en https://repository.eafit.edu. co/bitstream/handle/10784/944/2008_15_Marleny_ Cardona.pdf? sequence $=1$ \&isAllowed $=y$

Inadem (2017). 10 emprendedoras sociales que nos inspira. Instituto Nacional del Emprendedor, 27 de marzo. Recuperado el 1 de junio de 2018, de https:/www. inadem.gob.mx/10-emprendedoras-sociales-que-nosinspira/

Marulanda, F. y Morales, S. (2016). Entorno y motivaciones para emprender. Revista Escuela de Administración y Negocios, 81, 12-28. https://doi. org/10.21158/01208160.n81.2016.1556

Misión Planeta (2016). Misión Planeta. Recuperado el 1 de junio de 2018, de http://www.misionplaneta.org/

Moreira, P. y Urriolagoitia, L. (2011). El emprendimiento social. Revista Española del Tercer Sector, 17, 17-40.

Munster, B. (2017). La sostenibilidad de los emprendimientos solidarios de mujeres: Los desafíos para las políticas públicas. Economía y Desarrollo, 158(1), 91-105.

Naranjo, O. (2015). Propuesta de un modelo de negocios desde el emprendimiento social. Apuntes Universitarios. Revista de Investigación, 5(1), 35-50.

Nikulin, C., Viveros, P., Dorochesi, M., Crespo, A. y Lay, P. (2017). Metodología para el análisis de problemas y limitaciones en emprendimientos universitarios. Innovar. Revista de Ciencias Administrativas y Sociales, 27(63), 91-105.

Ocampo, D. (2016). El emprendimiento social en la formación integral. Revista Escuela de Administración de Negocios, 81, 175-190. https://doi. org/10.21158/01208160.n81.2016.1554

Oliver, A., Galiana, L. y Gutiérrez, M. (2016). Diagnóstico y políticas de promoción del emprendimiento en estudiantes. Anales de Psicología. 32(1), 183-189. https:// doi.org/10.6018/analesps.32.1.186681

Ortiz, P. (2017). El discurso sobre el emprendimiento de la mujer desde una perspectiva de género. Vivat Academia, 140, 115-129. https://doi.org/10.15178/ va.2017.140.115-129

Padilla, M., Quispe, A. L., Nogueira, D. y Hernández, A. (2016). Diagnóstico y perpecticas de fomento del emprendimiento como instrumento de desarrollo. Ingeniería Industrial, 37(1), 91-103.

Palacios, G. (2010). Emprendimiento social: Integrando a los excluidos en el ámbito rural. Revista de Ciencias Sociales, 16(4), 579-590.

Protrash (2017). Acerca de nosotros. ProTrash. Recuperado el 1 de junio de 2018 de https:/www.protrashco.com/ copia-de-home

Rodríguez, G. (2016). Educación informal en emprendimiento y creatividad en escuelas innovadoras. Opción, 32(12), 425-443.

Romero, A. y Milone, M. (2016). El emprendimiento en España: Intención emprendedora, motivaciones y obstáculos. Journal of Globalization, Competitiveness \& Governability. 10(1), 95-109.

Romero, J., Hernández, L., Gutiérrez, J. y Portillo, R. (2017). Factores contextuales que influyen en el emprendimiento de empresas familiares en Venezuela. Opción, 33(83), 492-515.

Contabilidad y Negocios (13) 26, 2018 / ISSN 1992-1896 
Sáenz, N. y López, A. (2015). Las competencias de emprendimiento social, COEMS: Aproximación a través de programas de formación universitaria en Iberoamérica. Revesco. Revista de Estudios Cooperativos, 119, $159-182$.

Sanabria, S. y Hurtado, E. (2018). Emprendimiento verde en torno a la conservación y recuperación del adua y sus espacios en Bogotá y Medellín (Colombia). Revista de la Facultad de Ciencias Económicas: Investigación y Reflexión, 26(1), 93-111.

Sarape Social (2016). Sarape Social. Ideas con Causa. Recuperado el 01 de junio de 2018, de http://sarapesocial. com/nosotros/

Sepúlveda, C. y Gutiérrez, R. (2016). Sostenibilidad de los emprendimientos: Un análisis de los factores determinantes. Revista Venezolana de Gerencia, 21(73), 33-49.

Stewart, K. (2002). Formal business planning and small business success: A survey of small business with and international focus. Journal of American Academy of Business, 2(1), 42-46.
Tu Ola (2016). Nuestra misión. Tu Ola. Recuperado el 1 de junio de 2018 de https://tuola.mx/

Vásquez, A. y Dávila, M. (2008). Emprendimiento social. Revisión de literatura. Estudios Gerenciales, 24(109), 105-125. https://doi.org/10.1016/S01235923(08)70055-X

Vázquez, J. C. (2017). Proyectos integrales de emprendimiento con sentido humano. Observatorio de Innovación Educativa, 25 de setiembre. Recuperado el 4 de junio de 2018, de https://observatorio.itesm. mx/edu-bits-blog/2017/9/25/proyectos-integrales-deemprendimiento-con-sentido-humano

Vega, J. y Mera, C. (2016). Modelo de formación en emprendimiento social para instituciones de educación superior en Colombia. Revista Escuela de Administración de Negocios, 81, 29-43. https://doi. org/10.21158/01208160.n81.2016.1563

Fecha de recepción: 12 de junio de 2018 Fecha de aceptación: 28 de agosto de 2018 Correspondencia: jcvazquezp@itesm.mx 


\section{Jorge Humberto Piedra Luna}

Estudiante del décimo semestre de la carrera de Contabilidad y Auditoría en la Unidad Académica de Ciencias Empresariales. Universidad Técnica de Machala.

\section{Nydia Marcela Reyes Maldonado}

Doctora en Contabilidad y Finanzas de las Organizaciones por la Universidad de Zaragoza de España. Magíster en Contabilidad y Finanzas de las Organizaciones por la Universidad de Zaragoza de Espańa. Contadora pública por la Universidad Autónoma de Bucaramanga. Docente del Programa Contaduría Pública de la Facultad de Ciencias Económicas, Administrativas y Contables de la Universidad Autónoma de Bucaramanga. Directora del Grupo de Investigación en Contabilidad y Finanzas - INVESCONFIN.

\section{Fernando Chaparro García}

Doctor en Ciencias Económicas y Empresariales por la Universidad de Zaragoza de España. Maestría en Administración por la Universidad Autónoma de Bucaramanga - ITESM México. Maestría en Contabilidad Internacional por la Universidad de Zaragoza de España. Contador público por la Universidad Autónoma de Bucaramanga. Docente de la Universidad Autónoma de Bucaramanga, Facultad de Ciencias Económicas, Administrativas y Contables, Programa de Contaduría Pública

\section{Carlos Alberto Oyola Moreno}

Candidato a doctor en Educación Universidad de Baja California. Magíster en Ciencias de la Información del Instituto Tecnológico de Estudios Superiores de Monterrey México (ITESMX). Contador público por la Universidad de Ibagué e ingeniero de sistemas por la Universidad del Área Andina. Docente asociado en la Universidad Autónoma de Bucaramanga, Facultad de Ciencias Administrativas Económicas y Contables. Especialista en Revisoría Fiscal y Auditoría Externa, especialista en NIIF certificado.

\section{Thayse Moraes Elias}

Magíster en Contabilidad por la Universidade Federal de Santa Catarina. Bachiller en Ciencias Contables en Universidade Federal de Santa Catarina. Investigador en el Grupo de Gestão de Custos - GGC.

\section{Eduardo Augusto Borgert}

Bacharel em Ciências Contábeis, Universidade Federal de Santa Catarina. Aluno de Mestrado.

\section{Altair Borgert}

Doutor em Engenharia de Produçáo por la Universidade Federal de Santa Catarina. Mestre em Administração por la Universidade Federal de Santa Catarina. Bacharel em Ciências Contábeis por la Universidade Federal de Santa Catarina. Docente titular 
en la Universidade Federal de Santa Catarina Departamento de Ciências Contábeis. Professor do Programa de Pós-Graduação em Contabilidade da Universidade Federal de Santa Catarina.

\section{Rogério João Lunkes}

Doctor en Engenharia de Produçáo e Sistemas por la Universidade Federal de Santa Catarina. Master en Engenharia de Produção e Sistemas por la Universidade Federal de Santa Catarina. Graduación en Ciências Contábeis por la Universidade Federal de Santa Catarina. Profesor del Departamento de Ciências Contábeis - Universidade Federal de Santa Catarina. Professor Associado III.

\section{Yully Marcela Sepúlveda Alzate}

Magíster en Administración por la Universidad Nacional de Colombia, Sede Bogotá. Contadora pública por la Universidad Nacional de Colombia, Sede Bogotá. Docente de la Universidad Santo Tomás, Sede Bogotá. División de Ciencias Económicas y Administrativas, Facultad de Contaduría Pública.

\section{Gloria Milena Valero Zapata}

Magíster en Medio Ambiente y Desarrollo por la Universidad Nacional de Colombia, Sede Bogotá. Contadora pública por la Universidad Nacional de Colombia, Sede Bogotá. Docente en la Universidad Santo Tomás, Sede Bogotá. División de Ciencias Económicas y Administrativas, Facultad de Contaduría Pública. Coordinadora del Centro de Investigación de la Facultad de Contaduría Pública [CICOP].

\section{Martha Janeth Bonilla Gómez}

Contador público por la Fundación Universitaria de San Gil, Santander. Contadora pública independiente.

\section{Luis Ferruz Agudo}

Doctor en Contabilidad y Finanzas por la Universidad de Zaragoza (UNIZAR). Docente en la Facultad de Economía y Empresa, en el Departamento de Contabilidad y Finanzas.

\section{Daniel Knebel Baggio}

Doctor en Contabilidad y Finanzas por la Universidad de Zaragoza (UNIZAR). Magíster en Contabilidad y Finanzas por la Universidad de Zaragoza (UNIZAR). Grado en Administración por la Universidade Regional do Noroeste do Estado do Rio Grande do Sul (UNIJUI). Docente en la Universidade Regional do Noroeste do Estado do Rio Grande do Sul (UNIJUI) y la Universidade Regional Integrada (URI)

\section{Gabriela Scheffer}

Grado en Administración por el IMED. Administradora de la empresa Ouro Scheffer.

\section{Bruna Faccin Camargo}

Doctoranda en Contabilidad y Finanzas por la Universidad de Zaragoza (UNIZAR). Máster en Desarrollo Regional por la Universidade Regional do Noroeste do Estado do Rio Grande do Sul. Grado en Contabilidad por la UNICRUZ. Docente en la Universidad Franciscana, en el Departamento de Contabilidad.

\section{Aloísio Rodrigues da Silva}

Doutorado em Contabilidade pela Universidad Nacional de Rosário - Argentina. Mestrado em Ciências Contábeis pela Fundação Getúlio Vargas - RJ. Graduado em Ciências Contábeis pela Universidade Federal de Mato Grosso; Especialista em Contabilidade pela Universidade Federal de Mato Grosso. Docente na Universidade Federal de Mato Grosso 
- Câmpus Universitário de Rondonópolis - Departamento de Ciências Contábeis.

\section{Wellen Kayzi Moraes de Almeida e Silva}

Graduanda Ciências Contábeis pela Universidade Federal de Mato Grosso. Tem experiência na área de Administração, com ênfase em Ciências Contábeis.

\section{Sofía Inés Niveiros}

Doutorado em Engenharia de Produção pela Universidade Federal de Santa Catarina. Mestrado em Engenharia de Produçáo pela Universidade Federal de Santa Catarina. Possui graduação em Ciências Contábeis pela Universidade Federal de Santa Catarina, e pós-doutorado em Engenharia e Gestão do Conhecimento pela Universidade Federal de Santa Catarina. Professor titular da Universidade Federal de Mato Grosso.

\section{Laura Chávez Cáceres}

Licenciada en Administración de Empresas por la Universidad Católica San Pablo. Asistente de Región I Promperú OMRSO.

\section{Walter L. Arias Gallegos}

Doctor en Psicología por la Universidad Nacional de San Agustín. Maestro en Ciencias de la Educación con mención en Psicopedagogía Cognitiva por la Universidad Nacional Pedro Ruíz Gallo. Psicólogo por la Universidad Nacional de San Agustín. Profesor auxiliar en el Departamento de Psicología de la Universidad Católica San Pablo. Profesor auxiliar dedicado a tiempo completo en la UCSP con énfasis en investigación. Investigador acreditado por el Concytec (código 4916). Autor de seis libros y varios artículos de investigación. Diplomado en Gestión estratégica de recursos humanos y Gestión de sistemas integrados.

\section{José Carlos Vázquez Parra}

Doctorado en Estudios Humanísticos por el Tecnológico de Monterrey. Maestría en Educación por la Universidad del Valle de Atemajac. Licenciatura en Derecho y licenciatura en Psicología otorgadas por la Universidad del Valle de Atemajac. Profesor-investigador de la Escuela de Humanidades y Educación, Región Occidente, del Tecnológico de Monterrey. Miembro del Sistema Nacional de Investigadores (Nivel 1). 
Contabilidad y Negocios pretende promover y difundir la producción de artículos sobre temas de actualidad de las disciplinas de Administración y Contabilidad. Asimismo, aspira posicionarse como una revista de alto nivel académico, tanto para la comunidad universitaria como para el ámbito empresarial. Por ello, está dirigida a investigadores, estudiantes y profesionales relacionados con estas disciplinas.

Nuestro objetivo es que los lectores aprecien el contenido de los artículos como una fuente importante de consulta y referencia en su quehacer académico o empresarial. De igual modo, se espera que estos contribuyan al estudio y debate de los temas que nuestros colaboradores aportan en cada publicación.

Los artículos seleccionados y publicados deben ser de contenido original e inédito, acerca de temas de interés académico y/o profesional de las especialidades de Contabilidad, Administración, Economía y especialidades afines. Dichos artículos son evaluados por árbitros de las áreas correspondientes. La evaluación de los artículos se realiza bajo la modalidad de doble ciego, es decir los árbitros no conocen la identidad de los autores y, viceversa, los autores no conocen la identidad de los árbitros.
En cuanto a las citas bibliográficas de otros autores, estas deberán ser incluidas en los artículos mediante la norma de referencias parentéticas (apellido, año, página). Asimismo, se debe tener en cuenta que todo texto que sea citado en el contenido del artículo deberá figurar de manera obligatoria en la bibliografía.

Los artículos propuestos para su publicación deberán adecuarse a los siguientes lineamientos generales:

- Presentación en Microsoft Word.

- Formato A-4, espacio y medio, letra Arial 11.

- La extensión aproximada debe fluctuar entre quince (15) y veinte (20) páginas. Ello incluye gráficos y cuadros, los cuales deberán ser entregados, adicionalmente, en PowerPoint, Excel y otros similares que se hayan empleado en su elaboración.

- Resumen (200 palabras aproximadamente), palabras clave (4) y título en español e inglés.

- Citas y referencias bibliográficas debidamente especificadas de acuerdo a las pautas del manual de estilo que emplea el Fondo Editorial de la PUCP, el cual puede ser consultado en la siguiente dirección:

http://www.fondoeditorial.pucp.edu.pe/

Contabilidad y Negocios se publica dos veces al año, en julio y noviembre.

* El resumen o abstract debe tener en promedio 200 palabras (tamaño referencial, mas no determinante) y explicar de manera concisa y concreta el contenido total del artículo a fin de lograr un carácter descriptivo y/o informativo. Las palabras clave deben ser cuatro (4) en promedio en ambos idiomas. 
Contabilidady Negocios expects to promote and spread the production of articles about recent topics related to Administration and Accountancy disciplines, and it aspires to be a journal with high academic level, both for the university community as well as for the business field, that is why it is directed to researchers, students and professionals related to those disciplines.

Our objective is that our readers appreciate the content of the articles as an important consulting and reference source for academic or business work with the purpose of helping studies and discussing topics that our cooperators bring in every publication.

The selected and published articles must be original and unpublished, they must have academic interest and must be related to the fields of Accountancy, Administration, and Economy. These articles are evaluated by referees of the corresponding areas.

The evaluation of each article uses the modality of a double blind review, that means the referees do not know the identity of each author and so do not they authors respect to their referees.

Related to bibliography notes of other authors, these must be included in the articles using the law of parenthesis reference (last name, year, page). At the same time, we must consider that all kinds of texts mentioned in the article need to be published in the bibliography.

The articles presented for publication must respect the following conditions:

- Microsoft presentation

- A4 format, one and half space, font: Arial 11

- Among fifteen (15) to twenty (20) pages extend, including graphics and charts, they have to be handed additionally in Power Point, Excel and others used in its work.

- Abstract (200 words), keywords (4) and title in Spanish and English

- Footnote and bibliography references clearly mentioned, according to the steps used in the Register Style from PUCP, which can be checked in the electronic journal version, in the following address:

\section{http://www.fondoeditorial.pucp.edu.pe/}

Contabilidad y Negocios is published twice a year, in July and November.

\footnotetext{
* The summary or abstract should be on average 200 words (size reference, but not decisive) and explain in a concise and concrete way the entire contents of the article in order to achieve a descriptive and / or informative. Keywords must be four (4) on average in both languages.
} 


\section{ACTUALIDAD CONTABLE}

The future accounting in insurance companies: The International Financial Reporting Standard 17 -

Insurance contracts

Jorge Humberto Piedra Luna

Difficulties on the measurement of biological assets in Colombia

Nydia Marcela Reyes Maldonado, Fernando Chaparro García, Carlos Alberto Oyola Moreno

\section{CONTABILIDAD DE GESTIÓN}

Analysis of the composition of costs and expenses in different continents

Thayse Moraes Elias, Eduardo Augusto Borgert, Altair Borgert, Rogério João Lunkes

An analysis of the studies about sustainability reports in Latin America

Yully Marcela Sepúlveda Alzate, Gloria Milena Valero Zapata, Martha Janeth Bonilla Gómez

\section{FINANZAS}

Análisis del impacto de las crisis financieras en las ventas de joyas de la empresa Ouro Scheffer

Luis Ferruz Agudo, Daniel Knebel Baggio, Gabriela Scheffer, Bruna Faccin Camargo

\section{AUDITORÍA}

Continued professional education: An investigative analysis of its benefits in the view of the independent auditors registered at the CNAI and linked to the CRC-MT

Aloisio Rodrigues da Silva, Wellen Kayzi Moraes de Almeida e Silva, Sofia Ines Niveiros

\section{ADMINISTRACIÓN}

Relevance of managerial skills since Arequipenian manager's perception

Laura Chávez Cáceres, Walter L. Arias Gallegos

Elements for the integral valuation of social entrepreneurship projects. A tool for the training of entrepreneurs 
SE TERMINÓ DE IMPRIMIR EN LOS TALLERES GRÁFICOS DE

Tarea Asociación Gráfica Educativa

Psje. María Auxiliadora 156, Breña

Correo E.: TAREAGRAFICA@TAREAGRAFICA.COM

Teléfono: 332-3229 Fax: 424-1582

Se utilizaron CaraCteres

Adobe Garamond Pro en 11 puntos

PARA EL CUERPO DEL TEXTO

Diciembre 2018 Lima - Perú 